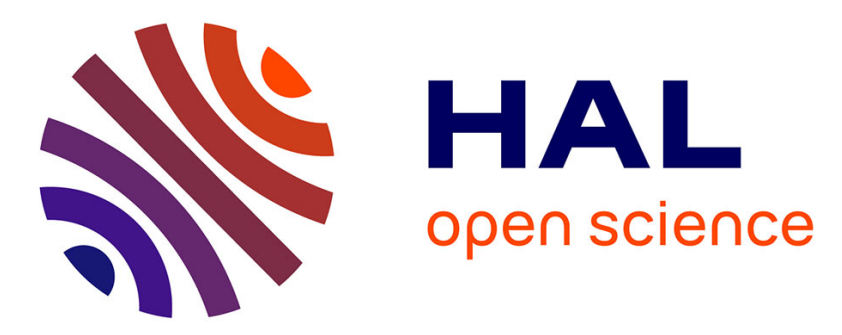

\title{
Les formes de scientificité dans la géographie enseignée L'évolution du discours scolaire sur les Pyrénées françaises (1872-1970)
}

Christine Vergnolle Mainar, Robert Sourp

\section{- To cite this version:}

Christine Vergnolle Mainar, Robert Sourp. Les formes de scientificité dans la géographie enseignée L'évolution du discours scolaire sur les Pyrénées françaises (1872-1970). L'Information géographique, 2004, 68, pp.173 - 190. 10.3406/ingeo.2004.2944 . hal-03166091

\section{HAL Id: hal-03166091 https://hal.science/hal-03166091}

Submitted on 11 Mar 2021

HAL is a multi-disciplinary open access archive for the deposit and dissemination of scientific research documents, whether they are published or not. The documents may come from teaching and research institutions in France or abroad, or from public or private research centers.
L'archive ouverte pluridisciplinaire HAL, est destinée au dépôt et à la diffusion de documents scientifiques de niveau recherche, publiés ou non, émanant des établissements d'enseignement et de recherche français ou étrangers, des laboratoires publics ou privés. 


\section{Les formes de scientificité dans la géographie enseignée L'évolution du discours scolaire sur les Pyrénées françaises (1872-1970) Christine Vergnolle-Mainar, Robert Sourp}

\section{Résumé}

L' analyse du discours scolaire sur les Pyrénées dans les manuels français de géographie, de 1872 aux années 1970, permet de mettre en évidence les liens que la géographie enseignée entretient avec la géographie savante. L'étude des notions et concepts introduits et de l'évolution des formes du raisonnement permet de constater que des processus de transposition didactique ont coexisté avec des formes d'autonomie de la géographie enseignée. Les rôles respectifs de l'un et de l'autre ont évolué au cours de la période étudiée de sorte que trois phases d'évolution sont mises en évidence : 1890, 1925 et les années 1960.

\section{Abstract}

The analysis of views expressed on Pyrenees in French geography text books, during the period from 1872 to the 1970 's, reveals the links between school geography and scientific geography. We study the notions which have been used and the evolution of reasoning, so that we can establish that didactic transposition and autonomy forms of school geography have existed simultaneously. The influence of each one has changed along the period studied and three evolution phases are identified : 1890, 1925 and the sixties.

\section{Citer ce document / Cite this document :}

Vergnolle-Mainar Christine, Sourp Robert. Les formes de scientificité dans la géographie enseignée. In: L'information géographique, volume $68, \mathrm{n}^{\circ} 2,2004$. pp. 173-190;

doi : https://doi.org/10.3406/ingeo.2004.2944

https://www.persee.fr/doc/ingeo_0020-0093_2004_num_68_2_2944

Fichier pdf généré le 29/03/2019 


\title{
Enseignement
}

\section{Les formes de scientificité dans la géographie enseignée}

L'évolution du discours scolaire sur les Pyrénées françaises (1872-1970)

\author{
Christine Vergnolle-Mainar et Robert Sourp \\ CeRF ( $: A$ 2182) et (iEOIDE (UMR 5602 ( NNRS) \\ IUFM de Midi-Pyrénées
}

\begin{abstract}
L'analyse du discours scolaire sur les Pyrénées dans les manuels français de géographie, de 1872 aux années 1970, permet de mettre en évidence les liens que la géographie enseignée entretient avec la géographie savante. L'étude des notions et concepts introduits et de l'évolution des formes du raisonnement permet de constater que des processus de transposition didactique ont coexisté avec des formes d'autonomie de la géographie enseignée. Les rôles respectifs de l'un et de l'autre ont évolué au cours de la période étudiée de sorte que trois phases d'évolution sont mises en évidence: 1890, 1925 et les années 1960.
\end{abstract}

Mots-clés : épistémologie, didactique, géographie scolaire, Pyrénées.

\begin{abstract}
The analysis of views expressed on Pyrenees in French geography text books, during the period from 1872 to the 1970's, reveals the links between school geography and scientific geography. We study the notions which have been used and the evolution of reasoning, so that we can establish that didactic transposition and autonomy forms of school geography have existed simultaneously. The influence of each one has changed along the period studied and three evolution phases are identified: 1890, 1925 and the sixties.
\end{abstract}

Key-words : epistemology, didactics, school geography, Pyrenees.

\section{Introduction}

Les Pyrénées françaises sont depuis un siècle et demi un objet scolaire bien identifié par les programmes et la totalité des manuels successifs. Cette continuité engage à analyser les rapports qu'une discipline, la géographie, entretient avec son double scolaire dans la durée. Le choix de cet objet correspond à une volonté de ne pas sélectionner un objet valorisé et par conséquent porteur d'implicites civiques forts ${ }^{1}$, mais au contraire un thème d'étude assez banal qui permette ainsi d'identifier l'influence en profondeur des divers facteurs de construction de la discipline scolaire. Le choix de la période de référence se rapporte à un cadre temporel où la

I. Sur un sujet valorisé. on peut lire: R. Sourp. "I es mutations des formes de causalité dans le raisonnement scolaire: l'exemple de la présentation de l'Furope en classe de quatrieme". L. Information géegraphique". vol. 65. sept. 2002 . p. $244-261$. 
géographie a gardé un statut assez stable, de 1872 où elle s'édifie en discipline scolaire dans le premier et le second degré, aux années 1970 qui la remettent en question et l'engagent dans une mutation conceptuelle et pédagogique. Le choix d'un corpus de manuels pour traiter de la géographie enseignée est également délibéré car c'est l'un des rares témoins des pratiques d'enseignement successives. À partir des manuels, il est possible de reconstruire celles-ci, car ils ont servi de référence, voire de modèle, tant pour l'enseignant que pour les élèves eux-mêmes. le corpus analysé couvre l'ensemble de la période et comprend les manuels principaux de l'enseignement primaire et secondaire, signés pour la plupart par des personnalités éminentes de la géographie (voir annexe).

Plusieurs auteurs ont cherché à repérer ce rapport entre les deux formes du savoir géographique dans la période envisagée ou pour une époque plus contemporaine. Dans un cadre similaire, le travail pionnier d'I. Lefort (1992) a cherché à clarifier le passage de la géographie de "matière culturelle à une discipline scientifique». Il fournit une base que l'on peut chercher à approfondir et à préciser en partant d'un objet d'enseignement sélectionné pour sa valeur d'exemple signifiant. Plus récemment, les recherches traitant de la période proche conduites par deux auteurs, D). Niclot et P. Clerc, nous offrent des cadres méthodologiques également transférables. Le premier montre que le savoir des manuels - ce qu'il nomme le "système-manuel" - correspond à une "alchimie" dosée au gré des auteurs, "de savoirs de sens commun, de savoirs issus de la tradition de la géographie scolaire, de faits d'actualité, d'objets scientifiques manuélisés et d'images du réel" (Niclot, 1999). Le second, en découpant un objet de programme, les villes, démontre que "sous l'apparence d'un inventaire neutralisé du monde, la géographie scolaire construit un modèle culturel» (Clerc, 2002).

Le découpage pratiqué traditionnellement par la géographie scolaire entre 1870 et les années 1970 à partir des Pyrénées, en tant qu'objet stable défini comme une région naturelle, porte des formes successives de scientificité qu'il convient d'identifier et de repérer. Dans ce projet, l'induration et la diversification des constituants du discours sur des notions et des concepts de la géographie classique ainsi que la pénétration de schèmes causaux représentatifs peuvent servir d'outils pour en repérer les transformations et permettre d'établir une périodisation.

\section{L'évolution des contenus de la géographie scolaire sur les Pyrénées}

Le socle initial de la géographie des Pyrénées, telle qu'on l'enseigne dans les différents niveaux du primaire et du secondaire au milieu du xix' siècle, est très limité: une vision romantique de la montagne qui privilégie les vallées agrestes et une conception militaire car il s'agit d'une montagne frontalière. La chaine n'est vue que dans cette identité globale et sous la forme de nomenclatures, d'inventaires d'activités et de faits. Si étroite soit-elle, cette base constitue le point de départ d'une évolution arborescente dans laquelle la filiation d'une notion à une autre occupe une place importante (fig. 1).

La présentation des reliefs occupe une place centrale pendant toute la période étudiée, mais la façon de les aborder a considérablement évolué. Les manuels les plus 


\section{Fig. 1 : Émergence des notions et des concepts}

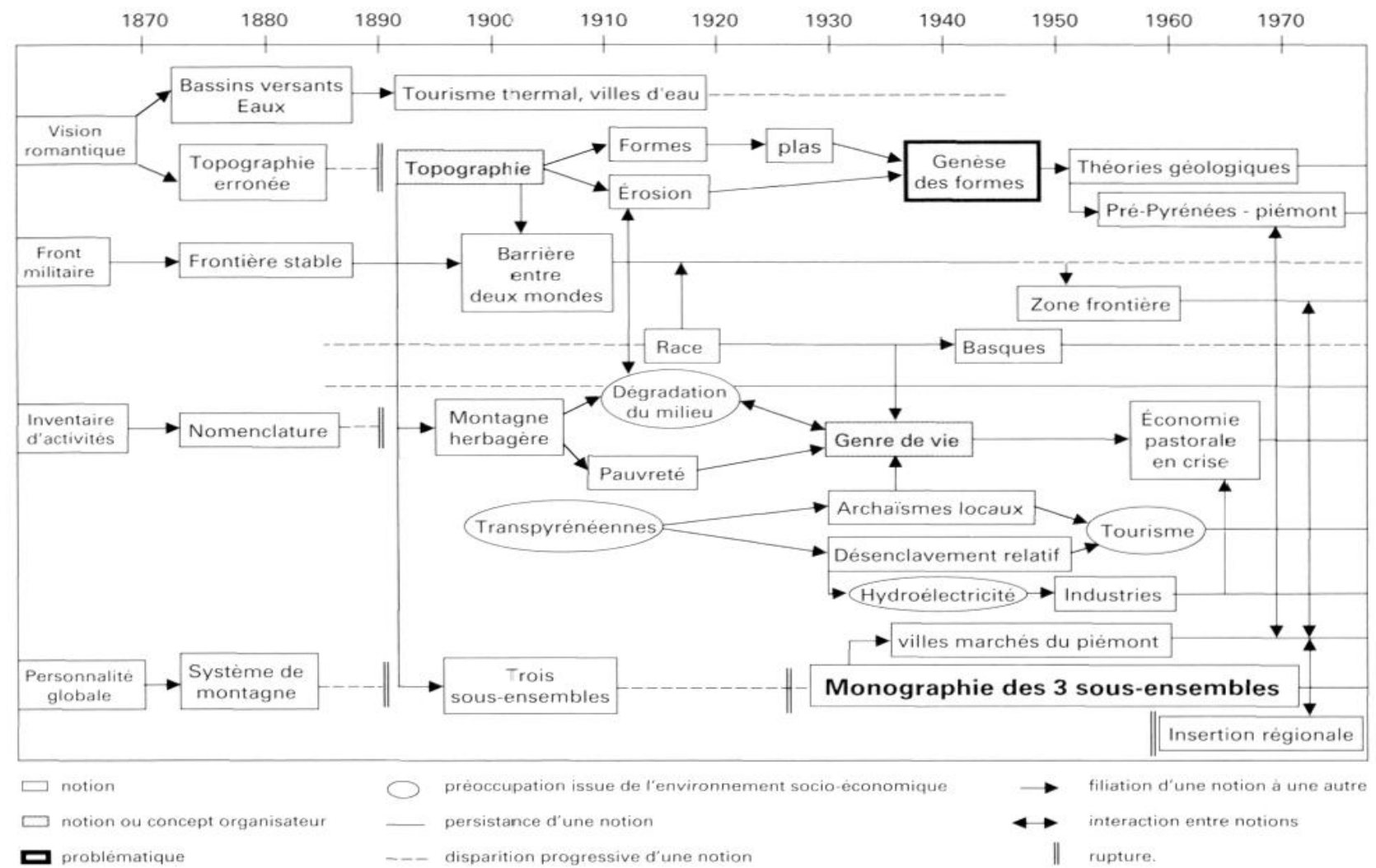

anciens adoptent une approche principalement centrée sur les bassins versants, relayant ainsi les conceptions développées au XVIIK siècle par Ph. Buache 2. Lorsque les zones hautes sont abordées, c'est souvent de façon approximative: la disposition des lignes de crêtes est comparée à une feuille de fougère et les monts ibériques sont considérés comme une ramification des Pyrénées. A partir des années 1880 , ces interprétations erronées cèdent progressivement la place à des descriptions topographiques plus précises et également plus justes s'appuyant sur des illustrations telles que des cartes du relief, des gravures de paysages et des blocs-diagrammes. Cette évolution débouche sur une comparaison avec la topographie alpine et surtout sur la mise en évidence de l'opposition entre le versant nord escarpé et le versant sud plus long et plus complexe. la pénétration des progrès dans la connaissance de la chaîne est ici notable et correspond à la reprise des travaux des pyrénéistes synthétisés par E. Reclus dans sa Géographie Universelle ${ }^{3}$.

Dans le prolongement de ces avancées, les manuels se font aussi l'écho des recherches sur les formes de relief et les processus d'érosion actuels et passés. Les formes "mûres" retiennent particulièrement l'attention, témoignant ainsi de la pénétration des interprétations que W.M. I)avis a développées a la fin du XIX" siècle. Dès 1910, certains manuels présentent les Pyrénées comme une "montagne adulte" aux pics trapus. I)ans les années 1920 , les "plas" sont identifiés en tant que formes topographiques et témoins des vestiges d'une pénéplaine. Ces sommets émoussés et ces replats perchés sont opposés à la "jeunesse" des formes des vallées. En 1937

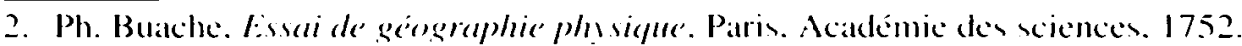

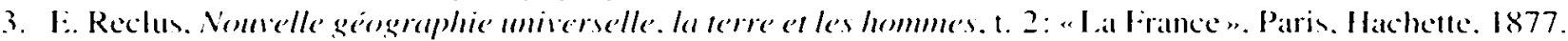


E. Baron expose explicitement le problème de la contradiction entre la coexistence de ces formes et les théories davisiennes. La question de la genèse des formes est dès lors posée et joue un rôle organisateur dans la présentation des connaissances. Les progrès des théories géologiques, largement relayées par les travaux des géomorphologues ${ }^{4}$, apportent des éléments de réponse à ce questionnement qui sont transposés dans les manuels des décennies suivantes. En liaison avec des présentations plus détaillées de la tectogenèse apparaît alors un intérêt croissant pour les pré-Pyrénées et le piémont. Dans ce contexte, les travaux de L. Goron ${ }^{5}$ et de F. Taillefer ${ }^{6}$ sont partiellement transférés par les auteurs de manuels.

L'ensemble de cette évolution témoigne d'une focalisation sur la géomorphologie et la géologie. Les autres aspects du milieu ne sont pas oubliés mais occupent une place secondaire et sont fortement mis en relation avec les formes du relief. Il en est ainsi de l'étagement et des oppositions climatiques, entre versants nord et sud d'une part et entre l'ouest et l'est d'autre part. Le milieu en tant que concept intégrateur n'est pas transféré dans les chapitres sur les Pyrénées. C'est au contraire la topographie qui joue un rôle organisateur.

\section{De la ligne de front a la marge}

La situation frontalière des Pyrénées est traitée de façon très différente dans le temps et est directement fonction du contexte international. La guerre de 1870 et toute la réflexion sur la place de l'enseignement de la géographie qu'elle a induite est à l'origine d'un point spécifique sur la frontière pyrénéenne dans toutes les études sur la France jusqu'en 1925. Au-delà, cet aspect n'est plus traité de façon systématique et n'est l'objet que de développements limités. Dans ces chapitres ou paragraphes, la frontière est très nettement vue sous un angle militaire, en tant que front ou ligne de défense. L'élévation de la chaîne et surtout la faible accessibilité des vallées et des cols sont considérées comme des éléments de défense «naturels». Mais ce «front du sud ou espagnol» est aussi présenté comme une frontière stable car elle est éloignée de la capitale et des zones de tension.

Malgré ce faible intérêt stratégique, l'étude des Pyrénées contribue à développer chez les élèves un sentiment de sécurité. La frontière est présentée comme étant bien gardée par les difficultés d'accès mais aussi par la loyauté de la population locale. La montagne est décrite comme une zone refuge dans laquelle les habitants se replient sur l'espace valléen et y développent à la fois une force de caractère et un attachement au sol. Il est sous-entendu qu'en cas de nécessité ces populations défendraient leur territoire et donc la patrie. Cette conception est particulièrement développée dans les manuels de l'école primaire et y est aussi souvent présentée de façon très déterministe. La mise en relation d'attitudes et même de valeurs morales avec les conditions de vie dans un milieu difficile rappelle vraisemblablement l'approche développée par E. Reclus (Debarbieux, 2001). Mais pour expliquer l'importance de cette thématique, il faut surtout prendre en considération le poids des finalités civiques qui pèsent sur l'enseignement de la géographie dans la perspective d'une revanche à la défaite de 1871 . Ces finalités, présentes pour tous les cycles d'enseignement, sont particulièrement affirmées pour l'école élémentaire

4. P. Birot. Recherches sta la morphogenèse des Prrénées orientales franco-espagnoles. Paris. Baillière et fils. 1937. 5. L. Goron, Lés pré-Pyrénées ariégeosisess et garonnaises, essai d'étude morphogénique d'une lisière de monta.gne. Toulouse, Privat, 1941.

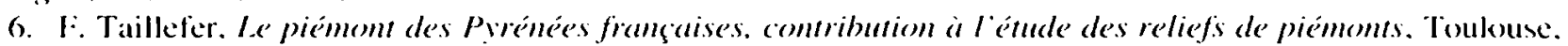
Privat, 1951. 
obligatoire. Cependant après la première guerre mondiale, les manuels présentent la population pyrénéenne davantage en tant que "race", avec une focalisation croissante pour les Basques "peuple à part" reprenant ainsi le qualificatif de J. Michelet'.

Tout au long de la période étudiée, les Pyrénées sont aussi associées à l'image de barrière. Les termes utilisés sont évocateurs: "muraille», "mur difficile à franchir", "vaste barrière", "énorme rempart», "obstacle»... Bien souvent placés en titre de paragraphe ou en introduction, ces termes sont renforcés par les gravures ou les photos qui viennent à l'appui. Celles-ci montrent principalement les Pyrénées centrales, vues de la plaine aquitaine (et notamment du boulevard des Pyrénées à Pau) ou d'avion pour les plus tardives. La chaîne apparaît comme "une crête dentelée", une "dent de scie» qui renforce l'impression d'une barrière infranchissable. Cette présentation contribue à caractériser les Pyrénées comme une montagne sans grande épaisseur et en marge du territoire français. La chaîne est une ligne de reliefs correspondant aussi à une ligne de défense. Ce n'est que dans les années 50 que la notion de zone frontière apparaîtra, témoignant de l'introduction tardive d'analyses de nature plus spatiale.

\section{lintregenres de vic et inventaire économique}

Les manuels de la seconde moitié du XIX siècle traitent les activités économiques sous la forme d'inventaires sans grandes liaisons avec les données relatives au milieu et parfois sans mentionner la présence des hommes. Seul le tourisme dans les villes thermales des Pyrénées centrales est mis en relation avec les ressources en eaux: sources minéralisées, cascades, rivières, lacs, etc. Cette approche, qui perdure jusqu'au milieu du $x^{c}$ siècle, est directement héritière de la vision rousseauïste de la montagne et dans sa forme reprend certains éléments de géographie de C. Malte Brun ${ }^{8}$. Parallèlement à cette vision romantique qui associe montagnes, ressources en eaux et richesse, se développe à la fin du XIX ${ }^{\complement}$ siècle un discours opposé qui a ses racines au début du XIX ${ }^{e}$ siècle (Laplace, 1995). La montagne est présentée à travers son activité pastorale et fait l'objet d'une comparaison négative avec les Alpes: ce sont le retard, le déclin, la pauvreté du massif pyrénéen qui sont mis en évidence. Les éleveurs sont aussi présentés comme responsables de la dégradation du couvert forestier et des problèmes érosifs qui lui sont consécutifs. Cette thématique, déjà présente dans certains manuels de la fin du XIX siècle, se développe fortement dans le courant de la première moitié du $\mathrm{xx}^{\mathrm{e}}$ siècle. Elle témoigne de la forte pénétration des interprétations développées par les forestiers et E. Reclus.

La façon de traiter des hommes et de leurs activités évolue profondément avec l'introduction du concept de genre de vie. Les paragraphes consacrés à la géographie humaine qui jusque-là étaient constitués de notions peu articulées entre elles, acquièrent un peu plus de cohérence. L'étude des genres de vie, d'abord présentés comme modes de vie, prend en compte les activités et principalement le pastoralisme, le peuplement et l'altérité des populations pyrénéennes, le milieu physique et sa dégradation par laction des hommes. Leur introduction peut cependant sembler tardive puisque, pour les Pyrénées, c'est le manuel de L. Gal-

7. J. Michelet. Tableau de la France. Paris. Les belles lettres. 1834.

8. C. Malte Brun et J.J.N. Huot. Préeis de la géographie universelle on description de toules le's parties du mont-

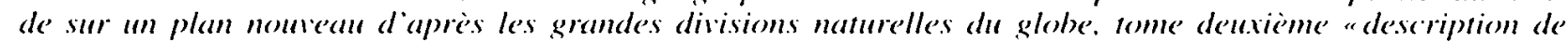
TEurope". Paris. Bureau des publications illustrées. 1840. 
louédec et F. Maurette de 1929 qui marque véritablement sa pénétration dans l'enseignement secondaire. Ce constat vient à l'appui de l'hypothèse (Mendibil, 1997 ) d'une pénétration plus lente du modèle vidalien dans les études de régions que pour la géographie générale. De ce fait ce sont moins les conceptions vidaliennes qui sont transposées que les avancées des recherches effectuées par l'École française de l'entre-deux-guerres. P. Vidal de la Blache n'est qu'exceptionnellement cité dans les références bibliographiques. Au contraire les auteurs de géographies régionales ${ }^{9}$ sont mentionnés et des extraits de leurs travaux sont parfois proposés en lecture. La transposition de ce concept n'est aussi que très partielle. Dans nombre d'ouvrages, tous niveaux confondus, elle se traduit moins par une réorganisation des notions présentées dans le texte que par le choix des illustrations: scènes de genre, maisons rurales, parmi lesquelles la maison basque occupe une place de choix.

Malgré le rôle organisateur de ce concept, la prédilection pour l'énumération des activités perdure jusqu'à la fin de la période étudiée. Elle se renforce même avec l'émergence de nouveaux secteurs économiques: échanges et ouverture de voies routières et ferroviaires transpyrénéennes, hydroélectricité et développement de nouvelles industries, tourisme automobile et d'hiver... Ces informations sont mises en relation entre elles et présentées comme une entrée dans la voie du progrès économique par opposition à l'économie pastorale traditionnelle en crise. Des photographies figuratives et de qualité illustrent ce modernisme. Mais les référénces scientifiques sont peu nombreuses "alors que la pénétration de préoccupations extérieures à la sphère de la géographic universitaire est nette: voies transpyrénéennes, tour de France, barrages d'EDF, etc. Ceci témoigne de la faible pénétration de l'approche développée par la nouvelle géographie et de l'influence croissante des arguments émanant des groupes de pression économiques.

La présentation des Pyrénées en tant que région a considérablement évolué en un siècle. Les premiers manuels étudiés ont une approche globale des Pyrénées considérées comme un "système de montagnes". L'identification de sous-ensembles dans les Pyrénées n'apparaît qu'à la fin du XIX siècle et est directement liée aux progrès dans la connaissance du milieu physique pyrénéen. La délimitation en trois sous-ensembles, Pyrénées occidentales, centrales et orientales, est consolidée par l'affirmation d'une approche régionale dans les programmes du secondaire de 1925. Au fil du temps elle tend à prendre de l'importance. La mise en évidence d'une identité pyrénéenne est reléguée au second plan voire occultée, y compris dans certains manuels de l'école primaire. Au milieu du xx' siècle, les auteurs tendent même à délimiter des sous-ensembles à l'intérieur de ces 3 grandes unités: l'avant-pays, les lignes de marchés du piémont, les premiers chaînons, sont alors l'objet de développements spécifiques.

La façon de traiter ces unités est très standardisée quel que soit le niveau d'enseignement. Il s'agit de la reproduction d'un plan à tiroirs dissociant fortement les aspects physiques et les données relatives à l'occupation humaine. Cette coupure entre géographie physique et géographie humaine est accentuée a partir de la

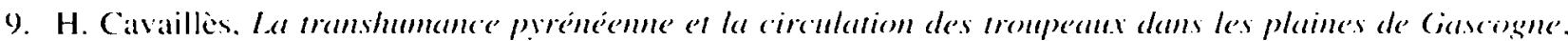

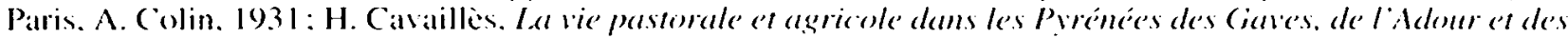

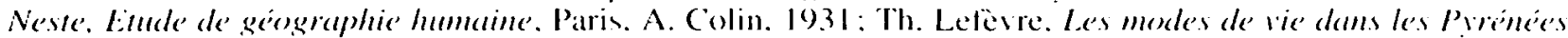

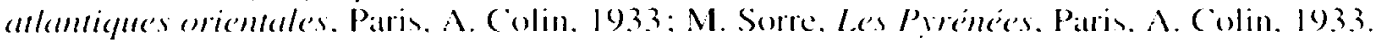

10. 1. Babonneatu. Lémergie électriche dems la région prénéenne. Toulouse. Privat. 
refonte des programmes du secondaire de 1902. Les manuels ne sont là que le reflet de la géographie universitaire comme en témoignent les deux géographies universelles, celle d'E. De Martonne " pour les données physiques et celle d'A. Demangeon '2 pour les activités des hommes. La présentation des Pyrénées aux différents niveaux d'enseignement doit aussi beaucoup a la vogue de la monographic régionale. Mais ce qui fait la spécificité de ce massif par rapport à d'autres régions françaises c'est à la fois la multiplicité des travaux de géographie régionale sur des portions de la chaine et l'absence d'une grande monographie. Il n'y a pas, pour les Pyrénées, l'équivalent des travaux de R. Blanchard ${ }^{13}$ sur les Alpes qui ont durablement servi de référence pour les contenus d'enseignement (David, 1984 et Debarbieux, 2001 ), d'où la dispersion des analyses.

Ce n'est que dans les années 1960 que des problématiques relatives à l'insertion des Pyrénées dans un espace régional plus vaste permettent de dépasser cet émiettement. En situant les Pyrénées par rapport aux grandes villes françaises et à la frontière espagnole, elles permettent d'envisager d'autres facteurs explicatifs que ceux liés au milieu et au caractère des habitants. Elles contribuent aussi à voir les relations des sous-ensembles les uns avec les autres. Ainsi, les zones basses sont présentées comme ouvertes, modernes et prospères et sont opposées a la montagne proprement dite, plus repliée sur elle-même et siège d'un déclin économique. Les manuels de $3^{\circ}$ et de $1^{\prime \prime}$ accompagnant les nouveaux programmes de 1963 sont ceux où le passage d'une approche monographique à une approche plus synthétique apparait le micux, avec toutefois un degré plus ou moins poussé selon les auteurs ou directeurs de collections qui pour la plupart sont des grands noms de la géographie de cette époque: Y. Lacoste, M. Baleste, M. Derruau, M. Le Lannou... Le transfert du progrès des connaissances sur la région concernée est moins net que dans la période précédente d'autant plus que les thèses sont désormais peu nombreuses et les synthèses se limitent à celle de G. Viers ${ }^{14}$ et F. Taillefer ${ }^{15}$. Par contre c'est l'évolution globale en géographie qui transparait dans ces études régionales de même que la diversification des approches.

La pénétration dans les manuels des notions et concepts produits par la recherche universitaire est done rapide quels que soient la période et le niveau d'enseignement considérés. Elle est directement liée à l'affirmation de l'École française de géographie. Mais il s'agit surtout de l'introduction de données factuelles assez indépendantes les unes des autres, la réorganisation des contenus d'enseignement autour de quelques notions clés n'étant au contraire que partielle et tardive. C'est en particulier le cas des genres de vie qui tendent a jouer un rôle fédérateur au moment même où la géographie enseignée se nourrit de plus en plus de sources dispersées et extérieures à la géographie (géologie, monde économique...). Ces notions, dont l'origine et le statut dans le discours scolaire sont très différents, constituent néanmoins le matériel sur lequel s'appuie la construction d'un raisonnement géographique adapté aux finalités de l'école.

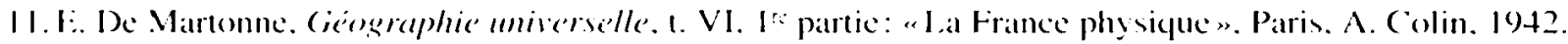

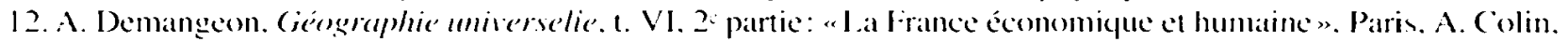
1946.

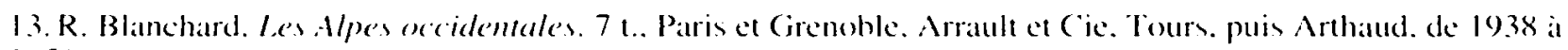
1956.

14. G. Viers. Les Pyénces. Paris. PLF. coll. Que Sais-Je". n 995. 1966.

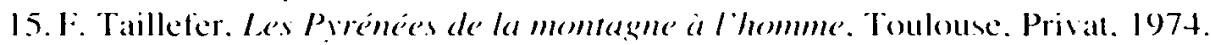

L'information géographique $-n^{\circ} 2,2004$ 


\section{Le raisonnement appliqué aux Pyrénées en tant qu'objet scolaire}

Objet d'étude bien individualisé en tant que région naturelle, les Pyrénées dans les manuels sont, comme nous l'avons déjà vu, décrites davantage dans leur différenciation topographique, géologique, bioclimatique et humaine que dans leur personnalité globale. Or, c'est cette dernière, prise en compte le plus souvent par la définition préalable de "traits généraux", qui construit l'argumentation destinée à la justifier. Les auteurs s'engagent rarement, avant les Instructions Officielles de 1925, dans des configurations explicatives. Lorsqu'elles apparaissent ensuite, les enchaînements causaux mis en œuvre dans le raisonnement peuvent être analysés comparativement avec le discours scientifique. Nous nous poserons donc deux questions: celle de la diffusion du raisonnement scientifique dans le texte des manuels et celle des formes de l'argumentation qui en sont issues et que contient le discours scolaire.

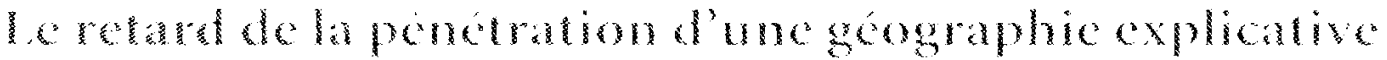

En écho aux mutations scientifiques apportées par l'arrivée de ce que l'on a appelé l'École française de géographie, les schèmes causaux qui en procèdent devraient apparaître dans le texte des manuels, au moins à partir de 1902, avec la rupture qu'apportent alors les programmes. Cette époque correspond à la parution du Tableau de la Géographie de la France ${ }^{16}$ et devrait donc marquer une mutation du discours explicatif scolaire. Pourtant, si on le compare au modèle supposé, l'écart du discours scolaire est considérable, traduisant une résistance du texte descriptif à la mise en place d'un raisonnement explicatif qui n'apparaît que beaucoup plus tard, dans les années 1925-1930. Cette résistance peut être renvoyée au lissage du texte des Instructions de 1902, peu explicite à cet égard et à l'imprégnation de la plupart des auteurs de la finalité de mémorisation de la discipline.

Dans le Tableau, comme l'a récemment montré D. Loi (2000), la causalité s'organise par convergence pour expliquer un fait particulier auquel une entité régionale doit sa singularité. Le particularisme pyrénéen est recherché dans un élément essentiellement historique, "la formation, au Moyen Âge, de cantons autonomes associant montagnes et plaines constitués en véritables États politiques». Ces derniers sont centrés sur la chaîne à ses deux extrémités ou sur les bassins hydrographiques ${ }^{17}$ en son centre. La matrice historique de ce découpage interne des Pyrénées est donc liée à un fait physique fondamental mais la combinaison causale procède de ces deux origines. En effet, «la plus continue des barrières» a d'abord servi de refuge pour les hommes, les amenant à se fixer précocement dans les hauts cantons, à développer leurs relations par les hauteurs et à concevoir une utilisation pastorale de la montagne qui associe les pâturages d'altitude et les basses vallées des deux côtés. Ce genre de vie, facteur de cohésion interne et externe, a contribué à l'apparition précoce d'associations d'éleveurs et l'émergence des petits États pyrénéens. La causalité vidalienne apparaît donc singulièrement complexe et cohérente dans sa structure idiographique (fig. 2).

16. P. Vidal de la Blache. Tablean de géographie de la France. Paris, Hachette, 1903.

17. Le plus cohérent est désigné par P. Vidal comme étant le Béarn. combinant montagne et plaine. "qui ne perdit pas sans regret $|\ldots|$ son autonomie dans l"unité française». 
Fig. 2 : Idiographie et raisonnement vidalien: une ossature rationnelle multicausale productrice d'un effet unique, le particularisme des Pyrénées

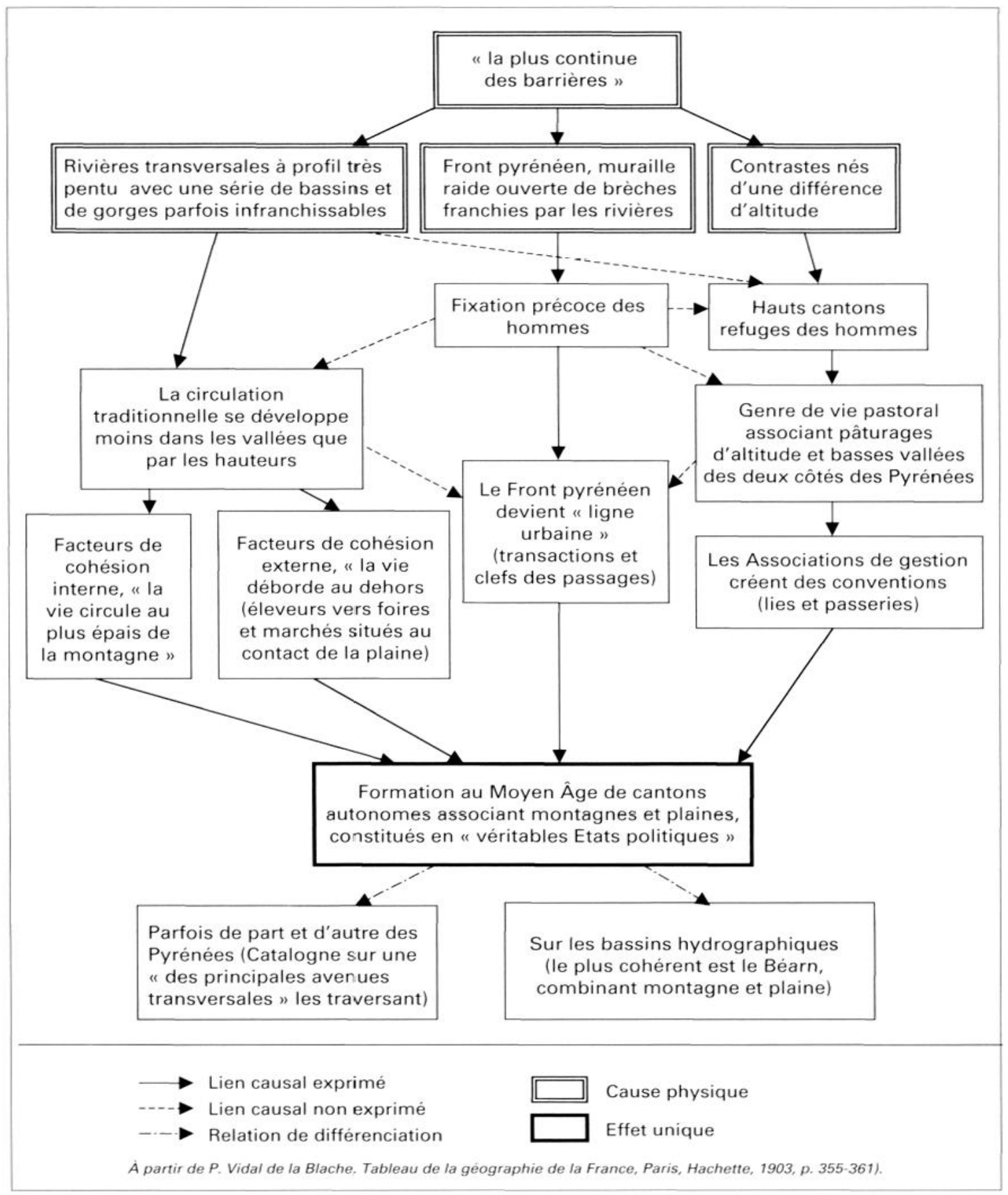

Si l'on met en regard de cette argumentation scientifique les manuels du temps, force est de constater qu'ils ne retiennent quasiment rien de ce schéma, soit qu'ils se refusent à entrer dans une structure explicative, soit qu'ils introduisent des linéarités causales limitées mais en relation avec les fondements de la causalité vidalienne, soit qu'ils mettent en cuvre des formes argumentatives distinctes. Dans le premier groupe, nous trouvons celui de Vidal de la Blache et Camena d'Almeida ${ }^{18}$, dont le texte de 1897, répété sans changement en 1902, est très descriptif et ne met aucunement en ouvre la structure causale construite peu après 18. P. Vidal de la Blache et P. Camena d Almeida. La France. classe de Première, Paris, A. Colin, 1897. p. 35.5-376. 
dans le Tableaut. Il faut attendre l'édition remaniée de $1910{ }^{19}$ pour que soient inclus des éléments limités de l'argumentation contenue dans cet ouvrage, mais sans la solide architecture causale qu'il y mettait en cuvre. Ces éléments n'y apparaissent d'ailleurs que dans la description régionale à propos des Pyrénées centrales et non dans la présentation des "traits généraux». Une simple mention, destinée à souligner l'originalité de cette partie de la montagne, y est faite à propos de la construction précoce "de petits États prospères et jaloux de leurs libertés". La distance entre le texte du Tableau et celui du manuel est significative du poids de la tradition narrative du discours scolaire et du refus de transposer ce qui fait le caractère innovant de l'explication contenu dans le modèle scientifique. Les autres manuels en usage avant 1914 sont aussi dépourvus d'ambition explicative.

Le manuel de F. Schrader et L. Gallouédec de 1903 apparaît isolé dans son amorce de raisonnement explicatif mais celui-ci est fondé sur des bases différentes de celui du Tableau. Pour ces auteurs, l'opposition entre les versants nord et sud procède du rôle de barrière qui a engendré une incapacité des relations transversales ${ }^{20}$. Il en résulte une séparation "entre deux mondes entre lesquels la nature n'a point ménagé de relations». Il s’agit là d'une linéarité causale réduite qui a peu de lien avec la structure argumentative du Tableau. Le texte se poursuit sur une explication prenant pour base le concept de milieu. Le versant français est présenté comme "médiocrement favorable à l'homme: ses ressources végétales sont faibles et il est mal pourvu de richesses minérales». Contrairement au schéma vidalien, la tradition déterministe joue ici à plein, même si les auteurs reconnaissent qu' «au moyen d'une irrigation mieux entendue, on pourrait doubler ou tripler les produits du sol.»

\section{WHC}

À partir des Instructions Officielles de 1925, plus nettes dans leur infléchissement vers une géographie explicative et plus explicites à cet égard, la causalité dépliée dans les manuels est largement dominée par un déterminisme naturel peu en accord avec le modèle "possibiliste". Mais, si une meilleure transposition de l'argumentation s'engage dans le discours de la géographie universitaire, la place de l'explication est encore très réduite dans la plupart des manuels et les schèmes causaux, quand ils existent, y sont peu élaborés. Ainsi, le modèle explicatif du Tableau ne se diffuse jamais dans les ouvrages scolaires dans sa totalité. L. Gallouédec et F. Maurette ${ }^{21}$, auteurs du manuel le plus édité dans cette période, engagent leur argumentation sur les bases suivantes:

- Les Pyrénées forment une masse haute, continue, épaisse et complexe.

- De leur hauteur et de leur complexité résulte une opposition entre le climat, la végétation et les ressources des versants Nord et Sud.

- De leur épaisseur résulte non seulement la difficulté des échanges à travers le massif, mais à l'intérieur de ce massif, l'existence de groupes de population isolés, indépendants, qui ont su préserver de l'influence française ou espagnole la pureté

19. P. Vidal de la Blache et P. Camena d'Almeida, La France. Paris. A. Colin. 1910. p. 370.

20. F. Schrader et L. Ciallouédec. (jéographie de la France. classe de Première. Paris. Hachette. 1903, 540 p. Ces auteurs écrivent (p. 250): "sur la moitié de la longueur totale du systène. les versants ne peuvent conmuniquer que par de rares sentiers de mulets, impraticables durant six ou sept mois de l"année, ou par des pistes de piétons qui serpentent entre les rochers à travers les cassures ou les ondulations de la crête. De là le contraste frappant. ke divorec tranché que présentent les deux versants pyrénéens".

21. L. Gallouédec, F. Maurette, Géographie de la France, classe de Première. Paris. Hachette. 1929.547 p. 
Fig. 3 : Les schèmes causaux du discours scolaire appliqué aux Pyrénées: chaînes linéaires, déterminants naturels

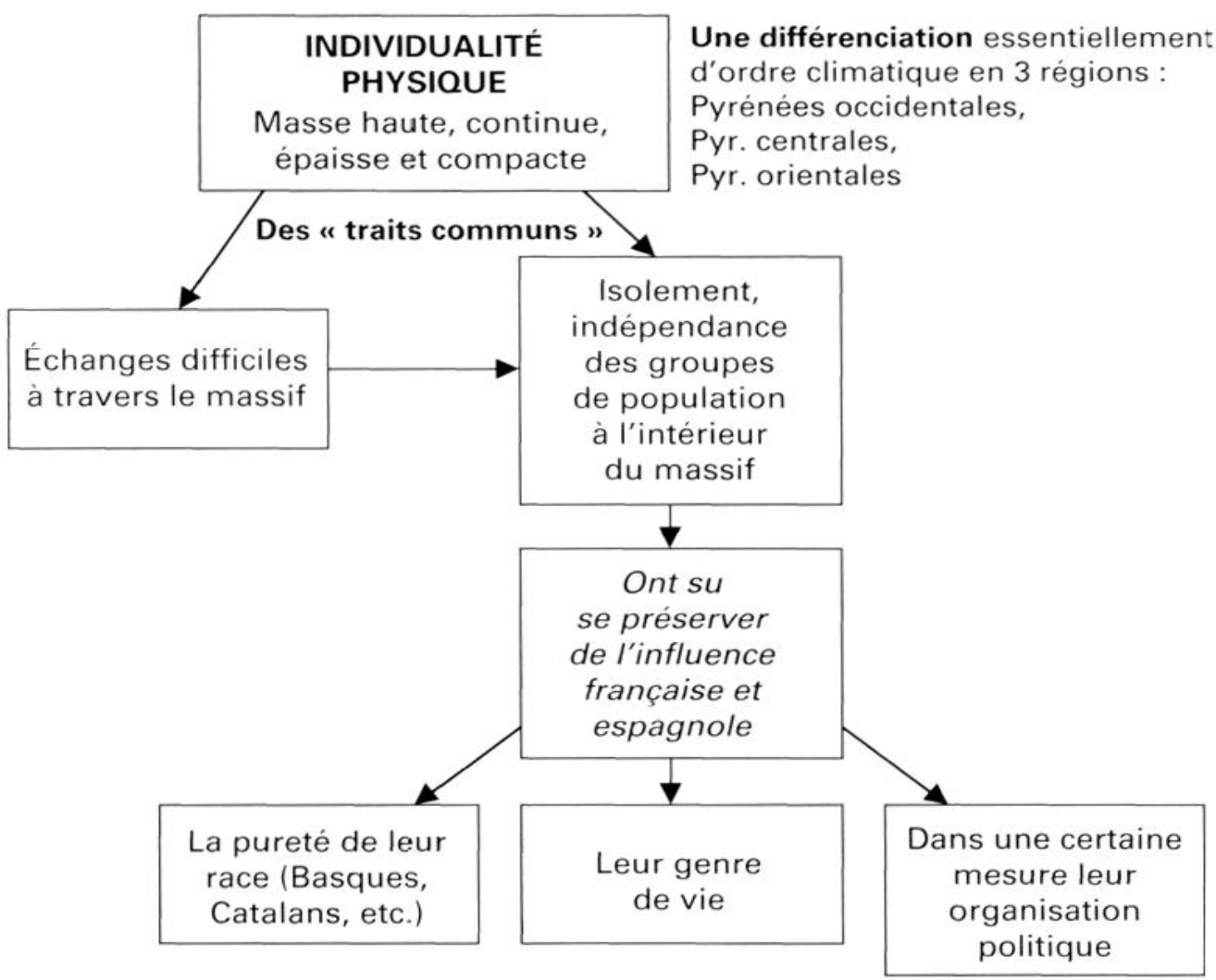

D'après L. Gallowedec, F. Maurette, Geographie de la France, classe de Premiere. Paris, Hachette, 1929, 547 p.

de leur race, leur genre de vie et même dans une certaine mesure, leur organisation politique.

Cet exemple est signifiant à deux titres, sur le type et la structure de la causalité scolaire la plus fréquemment pratiquée. I.e rapport causal s'établit dans un sens allant de la "Nature» vers les groupes humains, en relations linéaires binaires simples, et il ignore tout de la structure du discours de référence construite sur un modèle par convergence causale (fig. 3). Il y a alors quelque exagération à parler, comme le faisait I. Lefort (1992), de "vidalisme à l'école». Le discours scolaire est autonome par rapport au discours savant car celui-ci apparait aux auteurs trop complexe pour être transféré.

Lorsque le modèle scientifique propose des formes de raisonnement transférables au discours scolaire comme c'est le cas avec la notion de genre de vie, la transposition s'opère mieux. Après les travaux de R. Blanchard sur les Alpes françaises, ces dernières apparaissent comme l'archétype d'une utilisation réussie du milieu montagnard où les hommes ont su développer une organisation rationnelle des ressources dans le cadre du pastoralisme. Pour analyser d'autres massifs montagneux, le raisonnement se construit désormais de manière comparative. Le particularisme pyrénéen n'est vu alors que comme l'indice d'un retard économique dû à une maîtrise insuffisante du milieu. Expliquer cet écart conduit $M$. Auzelet (1931) à surdéterminer la part du milieu physique qui rend plus difficile l'exploitation des Pyrénées, du fait de l'insuffisance du travail de l'érosion fluviale ! fig. 4). Le discours scolaire s'en nourrit alors, car les schèmes causaux proposés entrent dans 
Fig. 4 : Raisonnement scientifique et raisonnement scolaire: les liens causaux dans le savoir de référence

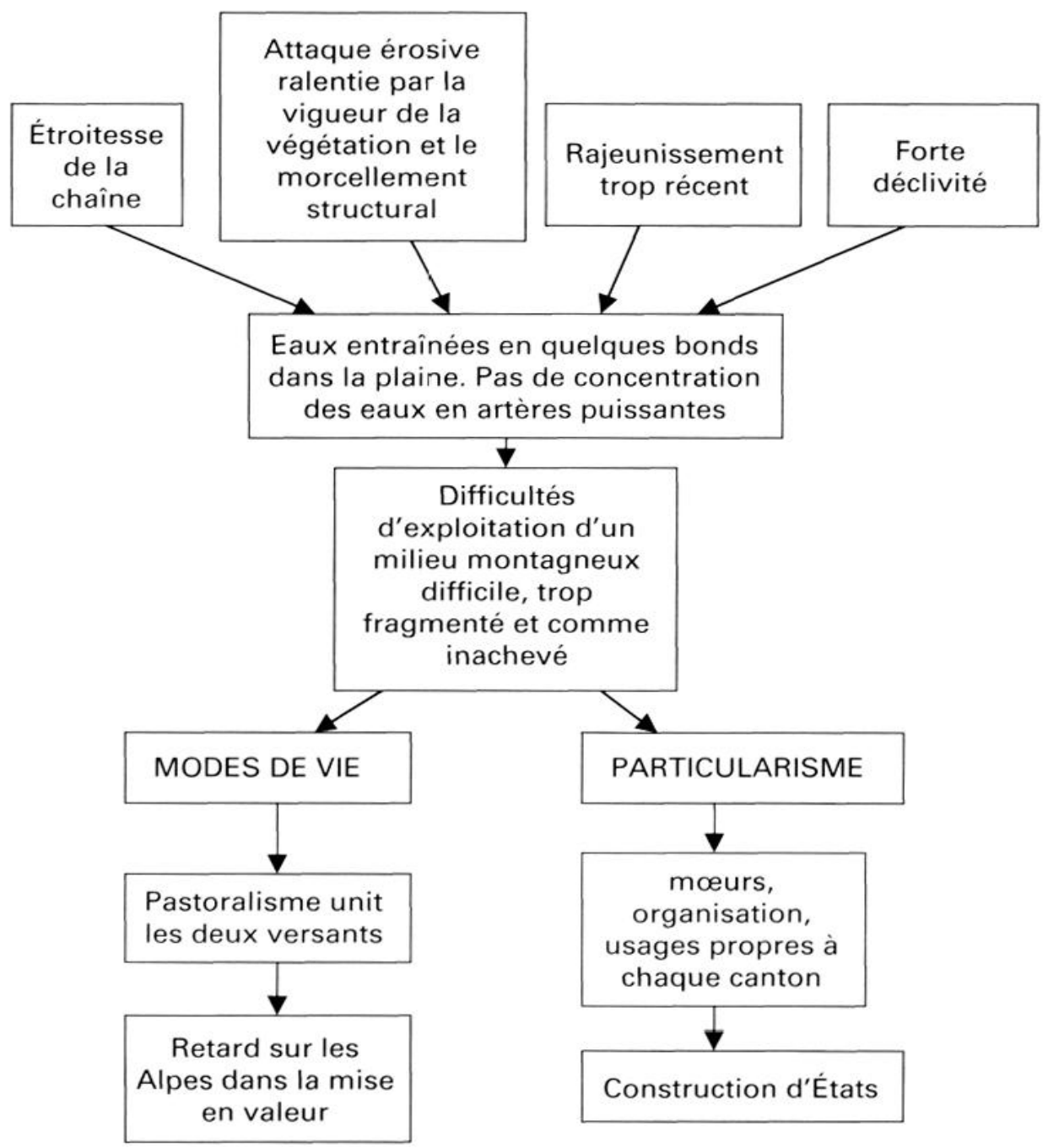

D'après M. Auzelet, "Les Pyrénées et le Bassin Aquitain",

in M. Allain (dir.), La Geographie universelle Quillet, tome'?

Paris, Editions Quillet, 1929, p. 369-363.

la tradition scolaire et surtout car ils apparaissent comme suffisamment simples pour pouvoir être transférés.

Le manuel d'H. Boucau (1935) suit des éléments de la chaîne causale ainsi définie en fondant sur le primat des contraintes physiques l'inachèvement et le morcellement de la montagne pyrénéenne, dus à une moindre action érosive, par opposition aux Alpes. Mais il intègre aussi vers l'aval, des éléments de raisonnement issus du Tableau pour expliquer le retard pyrénéen : le morcellement des vallées a conduit à la «formation au Moyen Âge de petits États, aujourd'hui petites cellules aux horizons limités» (fig. 5). Même si elle utilise des éléments du modèle vidalien, la structure argumentative de la géographie scolaire s'en écarte donc singulièrement...

On pourrait trouver chez la plupart des auteurs 22 contemporains de H. Boucau un raisonnement de ce type et un écart du même ordre. Mais celui-ci diffère dans

22. F. Baron. La France et ses colonices. Paris, Magnard. 1937. 342 p. : Cours M. Fallex: Cours Brunhes: Cours Demangeon; Cours Cholley: Cours Varon, etc. 
Fig. 5 : Les déterminations physiques dans le discours de géographie scolaire appliqué aux Pyrénées

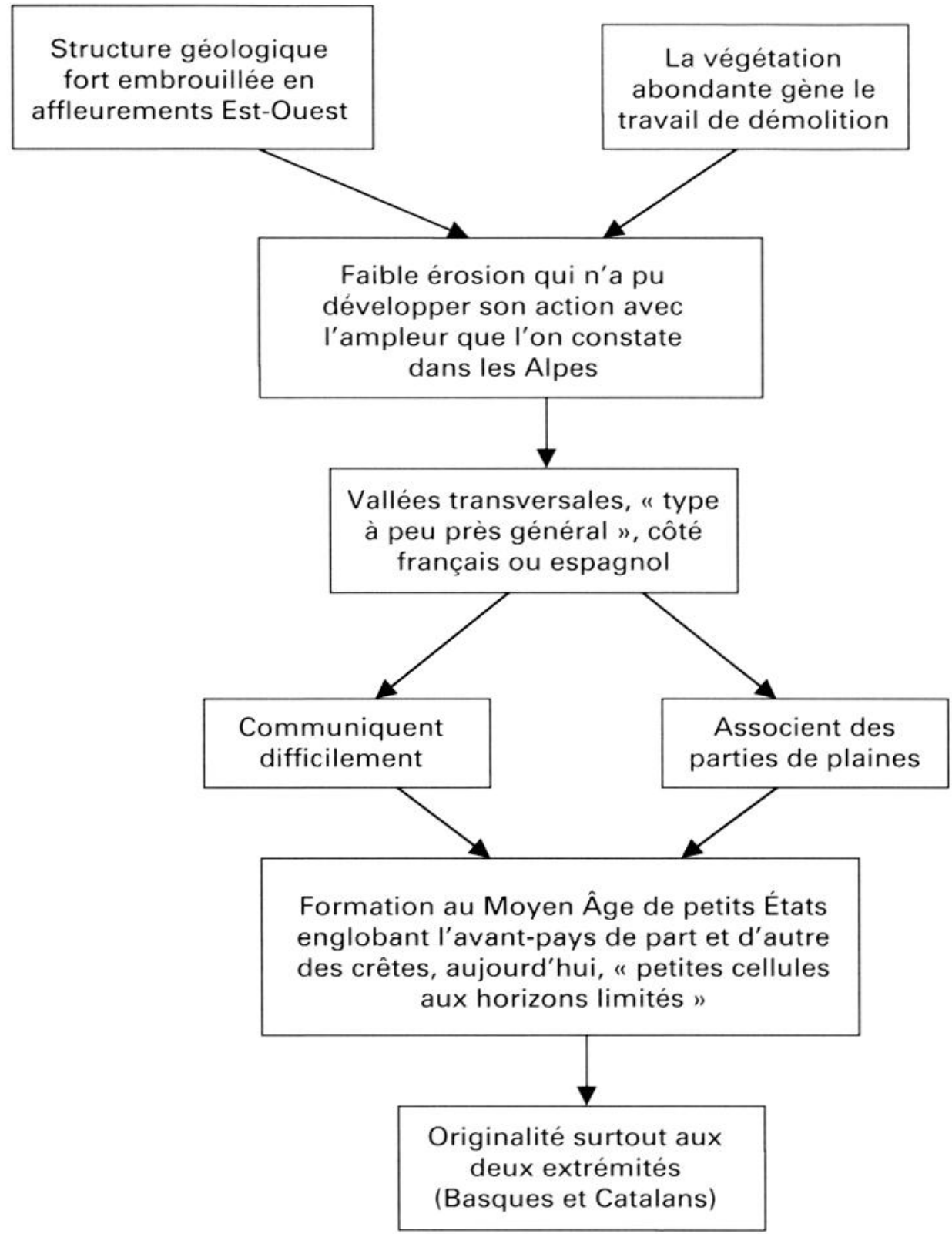

$\dot{A}$ partir de H. Boucau, La France et les colonies françaises, classe de Première, Paris, Hatier, coll. J. Brunhes, 1935.

ses fondements et dans sa structure car il présente des chaînes causales linéaires beaucoup plus réduites. Alors que le discours de la géographie classique déploie une causalité multiple produisant des effets convergents vers une résultante unique, la géographie des manuels, du fait de la réduction du texte, engage des chaines causales plus courtes, restreintes le plus souvent à un enchaînement d'éléments procédant de la nature. Surtout, les éléments implicites, relatifs à des problématiques sociales et/ou politiques, pèsent d'un poids très lourd dans l'argumentation pratiquée ${ }^{23}$. La diffusion du vidalisme apparaît donc singulièrement tardive et gauchie par rapport au modèle.

23. Pour les Pyrénées, la vision romantique véhicule une image d'exotisme et surtout d'archaisme qui reste présente dans l’argumentation. 
Tab. 1 : Bases du raisonnement des manuels dans la période 1960-1970

\begin{tabular}{|c|c|c|}
\hline $\begin{array}{l}\text { Manuels } \\
\text { (auteurs, éditeur, } \\
\text { date) }\end{array}$ & $\begin{array}{c}\text { Plan du chapitre } \\
\text { consacré aux Pyrénées }\end{array}$ & $\begin{array}{c}\text { Éléments } \\
\text { de l'argumentation }\end{array}$ \\
\hline $\begin{array}{l}\text { J. Pelletier, } \\
\text { J. Virlojeux, } \\
\text { coll. M. Derruau, } \\
\text { Masson, } \\
1961 .\end{array}$ & $\begin{array}{l}\text { 1) Les caractères généraux des Pyr. (un relief } \\
\text { intermédiaire entre celui des Alpes et celui du } \\
\text { Massif Central: une montagne aux formes } \\
\text { lourdes; une montagne aux climats très } \\
\text { différents; une vie de montagne plus agricole } \\
\text { que vouée à l'élevage et peu industrielle). }\end{array}$ & $\begin{array}{l}\text { Vidalisme: formes, genres } \\
\text { de vie. } \\
\text { Écart au modèle alpin; } \\
\text { moindre développement. }\end{array}$ \\
\hline $\begin{array}{l}\text { P. Gourou et } \\
\text { L. Papy, } \\
\text { Hachette, } \\
1962 .\end{array}$ & $\begin{array}{l}\text { Les Pyr. et leur avant-pays. } \\
\text { La barrière pyr. et son piémont. } \\
\text { La vie humaine dans les Pyr. (La vie rurale et } \\
\text { pastorale; les nouvelles activités). } \\
\text { La vie humaine dans l'avant-pays. }\end{array}$ & $\begin{array}{l}\text { Région géographique; } \\
\text { compartimentage; genres } \\
\text { de vie; transformation } \\
\text { des activités. }\end{array}$ \\
\hline $\begin{array}{l}\text { J. Madre, } \\
\text { coll. Baron, } \\
\text { Magnard, } \\
1963 .\end{array}$ & $\begin{array}{l}\text { 1) L'obstacle pyr. (Les Pyr sont beaucoup plus } \\
\text { massives que les Alpes; les Pyr. élevées avant } \\
\text { les Alpes puis ruinées par l'érosion, ont été } \\
\text { redressées en masse à la fin du tertiaire; les } \\
\text { rivières n'ont créé que de courtes vallées trans- } \\
\text { versales, étroits couloirs où les populations } \\
\text { vivent isolées). } \\
\text { 2) Les trois grandes régions pyr. }\end{array}$ & $\begin{array}{l}\text { Écart aux Alpes («pas de } \\
\text { quadrillage des vallées qui } \\
\text { fait la fortune des Alpes»); } \\
\text { Isolement, morcellement. }\end{array}$ \\
\hline $\begin{array}{l}\text { Gugliemo, } \\
\text { Lacoste, Ozouf, } \\
\text { Nathan, } \\
1963 \text {. }\end{array}$ & $\begin{array}{l}\text { Les Pyr, une montagne très différente des } \\
\text { Alpes. Une montagne massive aux formes } \\
\text { lourdes; des vallées courtes et étroites; une } \\
\text { montagne moins transformée que les Alpes. }\end{array}$ & $\begin{array}{l}\text { Écart aux Alpes, transfor- } \\
\text { mations limitées, condi- } \\
\text { tions physiques médiocres }\end{array}$ \\
\hline $\begin{array}{l}\text { R. Blanchon, } \\
\text { A. Labaste, } \\
\text { R. Oudin, } \\
\text { A. Colin, } \\
1963 .\end{array}$ & $\begin{array}{l}\text { 1) Une chaîne étirée en longueur, rigide et mas- } \\
\text { sive (un simple liseré montagneux mais diffi- } \\
\text { cile à franchir; une chaîne plus ancienne que } \\
\text { les Alpes; de grands contrastes climatiques) } \\
\text { 2) Une économie moins transformée que celle } \\
\text { des Alpes (la vie rurale reste archaïque; } \\
\text { l’hydro-électricité a rénové la tradition } \\
\text { industrielle; malgré des difficultés de la circu- } \\
\text { lation, le tourisme se développe; la montagne } \\
\text { se dépeuple cependant). } \\
\text { 3) Les contrastes régionaux. }\end{array}$ & $\begin{array}{l}\text { Écart aux Alpes, transfor- } \\
\text { mations moindres; } \\
\text { rénovation, développe- } \\
\text { ment. }\end{array}$ \\
\hline $\begin{array}{l}\text { coll Bertrand, } \\
\text { Delagrave, } 1963 .\end{array}$ & $\begin{array}{l}\text { Présentation des Pyr. Les conditions physi- } \\
\text { ques. Les activités. } \\
\text { Les aspects régionaux. }\end{array}$ & $\begin{array}{l}\text { Région géographique, } \\
\text { genres de vie. }\end{array}$ \\
\hline $\begin{array}{l}\text { coll. M. } \\
\text { Le Lannou, } \\
\text { Bordas, } \\
1966 .\end{array}$ & $\begin{array}{l}\text { 1) Le cadre physique. } \\
\text { 2) La vie rurale et pastorale. } \\
\text { 3) Formes anciennes et modernes de l'indus- } \\
\text { trie. }\end{array}$ & $\begin{array}{l}\text { Genres de vie (archaïsme } \\
\text { et isolement). Modernisa- } \\
\text { tion. }\end{array}$ \\
\hline
\end{tabular}


Les manuels de cette période apparaissent singulièrement paradoxaux dans la mise en ceuvre du raisonnement (tab. 1). D'une part, ils affichent avec constance la doxa de la géographie classique, en la rigidifiant dans une coupure marquée entre géographie physique et géographie humaine, mais sans que nécessairement la seconde procède de la première. Ils accompagnent alors par là l'évolution parallèle de la géographie universitaire. D'autre part, il y a glissement d'un raisonnement axé strictement sur le milieu à un raisonnement construit sur l'entre-deux de l'archaïsme des structures économiques de la production et de la modernité de l'intensification et de la diversification des activités. La notion de région considérée d'abord comme "l'harmonie entre la nature et les réalisations humaines" ${ }^{-4}$, sert ensuite de modèle comparatif pour l'évaluation d'autres ensembles régionaux similaires. L'écart à l'archétype est alors repéré avec précision dans les performances économiques respectives et l'adaptation à l'évolution des techniques.

Dans les ouvrages scolaires, le socle du vidalisme subsiste comme cadre du raisonnement mais en juxtaposant, dans leur texte, l'analyse du "cadre physique » et celle de la "mise en valeur humaine". Les programmes considérant les Pyrénées comme une région naturelle, la plupart des auteurs sur-valorisent cette base. J. Pelletier et J. Virlojeux (Masson, 1961 ) et J. Madre, dans la "Nouvelle collection Baron" (Bordas, 1963), lui accordent une part importante de leurs développements respectifs, près des trois quarts pour les premiers, près des deux tiers pour le second. La sacralisation de la coupure entre géographie physique et géographie humaine y manifeste surtout la rigidification du cadre dans lequel s'exerce le raisonnement sous l'emprise de la tradition. Mais d'autres auteurs, en restant dans un moule identique, élargissent leur raisonnement en relation avec l'approfondissement de la théorisation sur la région géographique. S’interrogeant sur la réalité régionale pyrénéenne, le manuel de Delagrave (collection Bertrand, 1963) souligne que leur manque d'extension en fait une "simple toile de fond de l'Aquitaine" et ne leur permet pas de "constituer une unité économique indépendante". P. Gourou et L. Papy (Hachette, 1962), dans le même esprit, élargissent la base d'étude en intégrant "l'avant-pays" pyrénéen. La question du découpage et des fondements de la réalité régionale évoque alors les débats de la communauté scientifique sur les bases d'analyse et les contenus du concept 25 .

Mais la perspective reste fortement marquée par le raisonnement vidalien. La problématique globale qui donne sa cohérence à l'ensemble des raisonnements appliqués dans la géographie scolaire ${ }^{2 h}$ n'est certes plus celle de l'adaptation au milieu montagnard mais celle de l'optimalisation de sa maitrise. Ia comparaison avec le modèle alpin est particulièrement rémanente et générale dans l'ensemble du corpus. La problématique de l'archaïsme, du "retard" engage le raisonnement sur une base comparative essentiellement négative. Son axe est dans la transformation des activités, dans l'évolution des structures productives, dont on analyse le rythme et le bilan comme étant insuffisants. Ceci est vérifié pour la spécialisation

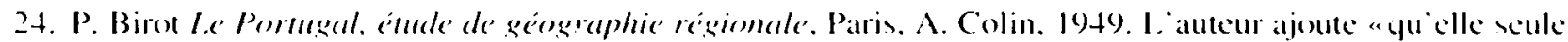
caractérise unc région au sens de Vidal de la Blachen.

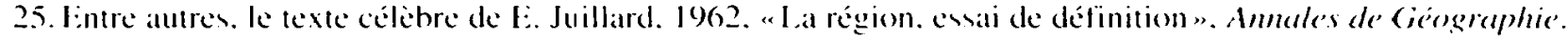
26. Nous asons sélectionné en priorité les ousrages de la classe de première produits a partir des I.0. de 196.3 qui domnent a létude régionale son extension maximale.
} 
agricole par la totalité des auteurs, ainsi que pour l'industric en cours de rénovation grâce au moteur de l'hydro-électricité (P. Gourou et L. Papy; Bertrand; R. Blanchon, A. Labaste et R. Oudin) ou pour l'essor du tourisme (Guglielmo, Lacoste, Ozouf, Bertrand).

Sur fond de péjoration de l'économie pyrénéenne, la problématique de la relation hommes-milieu qui structurait l'analyse de la géographie classique tend à être supplantée par celle du développement ${ }^{2 i}$, marquant les évolutions de la pensée géographique des années 1950-1970 vers les faits de production et vers des modèles archétypaux d'évolution des milieux. Mais cette évolution témoigne plus de l'émergence de nouveaux centres d'intérêt dans la géographie universitaire et dans les milieux économiques que de la pénétration de formes de raisonnement renouvelées. Le changement de paradigme disciplinaire que supposera l'apparition de "la nouvelle géographie" n'est nulle part perceptible dans le texte d'auteurs pourtant la plupart universitaires et engagés au même moment dans son émergence scientifique. La rigidité du cadre sur lequel les Instructions de 1963 les engagent, interdit ce transfert d'un "savoir scientifique" qui gagne en cohérence devenant ainsi un "savoir de référence", un modèle, "un savoir savant». Ce transfert ne sera amorcé que progressivement, et après 1985, par une recomposition de la géographie scolaire en adaptations successives.

\section{Conclusion: rythmes et moteurs de l'évolution}

L'enseignement de la géographie appliqué aux Pyrénées n'a pas suivi une évolution linéaire. Trois phases successives, initiées à partir des années 1890, 1925 et 1960 peuvent être mises en évidence.

Les années 1890 marquent l'émergence d'une géographie fondée sur des notions scientifiques. Tandis que les centres d'intérêts antérieurs sont peu à peu marginalisés, apparaît un réel souci de transfert de la recherche à l'enseignement et notamment un effort d'organisation des connaissances autour des progrès en matière de topographie. Cependant dans cette évolution, d'autres facteurs que les progrès scientifiques interviennent. On note surtout le rôle de l'institution scolaire qui assigne des finalités et fixe l'organisation de l'acquisition des connaissances. Dans ce sens les années 1890 marquent l'aboutissement d'une série de réformes des cursus scolaires et de redéfinition de la place relative de chaque discipline. Amorcées avec les directives Fourtoul de 1852, les réformes se sont accélérées à partir de 1872 (Marchand, 2001). Dans ce contexte, la géographie scolaire devient une discipline culturelle investie d'une mission de formation des futurs citoyens. Elle contribue à construire des référents communs à tous les Français et à développer un attachement à la patrie. Ceci pèse considérablement sur les programmes et sur la façon de conduire l'étude des différents points. La géographie scolaire a donc une marge d'autonomie par rapport à son homologue scientifique, ce qui explique que les Pyrénées soient avant tout présentées comme une barrière frontalière. Dans ce cadre, les nombreuses thématiques qui émergent au début du $\mathrm{xx}^{\mathrm{c}}$ siècle, en liaison avec les progrès de la recherche universitaire, ne génèrent pas de profondes réorganisations. La réforme de 1902 consacre certes l'influence des géographes

27. Bien que ce terme n’apparaisse pas toujours explicitement (présent seulement che\% J. Pelletier et J. Virlojeux). mais il est impliqué par d’autres termes comme ceux de "transformations limitées» (Ciugliemo. lacoste. (Onouf) de «transformations moindres" (R. Blanchon. A. Labaste et R. Oudin), de “transformations profondes» (P. Gourou et I.. Papy). 
universitaires dans la conception des programmes (Lefort, 1992) mais les formes de raisonnement different fortement entre géographie scientifique et géographie scolaire. De même les notions mises en évidence par la recherche scientifique sont intégrées assez rapidement dans les contenus d'enseignement mais elles viennent s'ajouter aux données déjà enseignées sans réelle recomposition des savoirs. Il s'agit donc plus d'un transfert que d'une transposition.

Après 1925 le changement est plus net. Les programmes consacrent l'approche régionale et introduisent plus nettement la géographie vidalienne. Cela se traduit par une percolation en profondeur du vidalisme. Le concept clé de genre de vie est transféré et contribue à une organisation de notions jusque-là présentées de façon très disjointe. Mais celte recomposition est limitée car les contenus d'enseignement véhiculent aussi nombre d'informations issues d'autres sources que la géographie universitaire, souvent mal reliées entre elles et peu structurées par les concepts scientifiques. Concernant les formes de raisonnement, on note également une meilleure transposition. Certes les schèmes causaux complexes développés par la géographie vidalienne pénètrent encore peu les contenus enseignés. Mais la structure de l'argumentation présentée par les manuels, qui est très linéaire, peut être considérée comme l'adaptation du discours universitaire au niveau des élèves et aux finalités de l'enseignement qui, même en lycée, ne vise pas à former des spécialistes. Ces formes de transposition sont cependant très tardives par rapport à l'affirmation de la géographie vidalienne. De ce fait elles sont moins liées à l'influence des textes fondateurs qu'au rôle des élèves de Paul Vidal de la Blache, tant dans les progrès de la recherche scientifique que dans l'écriture ou la coordination de manuels scolaires. L'analyse des contenus d'enseignement permet d'identifier là un «second vidalisme».

La dernière période d'évolution identifiable est celle des années 1960, dans le prolongement de la refonte des programmes en 1963. Dans cette période des trente glorieuses, la géographie enseignée n'est plus investie de finalités civiques aussi fortes qu'avant. L'objectif relatif à l'amour et à la défense de la patrie n'est plus d'actualité mais est relayé par la volonté d'accompagner l'engagement du pays dans la voie du modernisme. Les Pyrénées ne sont donc plus avant tout une frontière mais une région périphérique parmi d'autres. Cette portion de l'espace français est désormais bien connue scientifiquement et les manuels répercutent ces avancées. Mais la lacune est d'ordre méthodologique. L'accumulation de connaissances actualisées se fait au détriment de leur organisation autour de concepts forts et de raisonnements structurés. Cette carence est à mettre en relation avec le fait que le paradigme de l'école vidalienne n'est plus opérationnel et que celui proposé par la nouvelle géographie n'est pas encore transposé.

L'évolution du contenu de la géographie enseignée résulte donc de facteurs d'ordre scientifique, institutionnel et socio-économique. Dans un premier temps le cadrage par l'institution a joué un rôle majeur, puis le poids scientifique des chercheurs ainsi que l'influence du contexte socio-économique a pris de l'importance. Ceci confère à la géographie enseignée, quelle que soit l'époque considérée, une part d'autonomie par rapport à son homonyme universitaire qui se traduit par un transfert fidèle et rapide des notions mais une transposition sélective des modes de raisonnement et des concepts. 


\section{Bibliographie}

Clere P. (2002). Ia culture scolaire en géographie. Rennes. PUR. 185 p.

Debarbieux B. (20)1), «I es montagnes: représentations et constructions culturelles», in Y. Veyret (dir.), Les montagnes, discours et enjeux géographiques, Paris, SEDES, p. 35-45.

Debarbieux B. (2001). "La montagne: un objet géographique?". in Y. Veyret (dir.). Lees montagnes, discours et enjeux géographiques. Paris. SEDES. p. 11-34.

David J. (1984). "Images et stéréotypes d une montagne. les Alpes françaises dans les manuels de l'enseignement secondaire». Revere de Géographie Alpine, 72, n'2-3. p. $1.55-164$.

I aplace D. (1995), «I es géographes vidaliens face à l'altérité pyrénéenne». in Berdoulay

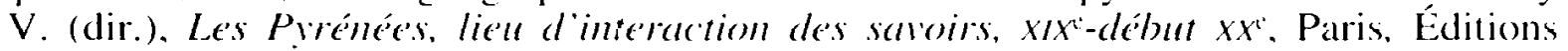
CTHS, p. 83-96.

I.efort I. (1992), La lettre et l'esprit, géographie scolaire et géographie savante en France. Paris, CNRS, $257 \mathrm{p}$.

Loi D. $(2000)$, "Caractères de la causalité vidalienne et objet de l'explication dans le tableau de la géographie de la France», in M.-C. Robic (dir.). Le tableau de géographie de la France de Paul Vidal de la Blache. Paris. Éditions du CTHS, p. 107-117.

Marchand Ph. (20)1), L'historie' et la géographie dans l'enseignement se'condaire. textes officiels, rome l: 1795-1914. Paris. INRP. $782 \mathrm{p}$.

Mendibil D. (1997). Texte's at images de liconographie de la France (de 1840 à 1990). essai d'iconologie géographique, thèse de doctorat, université de Paris 1.

Niclot D. (1999). Le's systemes manuels d'une discipline scolaire': les manuclis de géographie de la classe de seconde publiés de 1981 à 1996 . thèse de doctorat, université Paris VII.

Sourp R. (20(02). "Les mutations des formes de la causalité dans le raisonnement scolaire: l'exemple de la présentation de l'Europe en classe de 4' ". L'Information géographicque. vol. 65 . p. $244-261$.

\section{Tab. 2 : Annexe: corpus étudié}

\begin{tabular}{|c|c|c|}
\hline École élémentaire & $\begin{array}{c}\text { Enseignement } \\
\text { primaire supérieur }\end{array}$ & Enseignement secondaire \\
\hline $\begin{array}{l}\text { Cours préparatoire: } \\
\text { 1923: H. Lemonnier, F. } \\
\text { Schrader } \\
\text { Cours élémentaire: } \\
\text { 1881: H. Lemonnier, F. } \\
\text { Schrader, M. Dubois; } \\
\text { 1912: L. Lanier, C. } \\
\text { Rogeaux, A. Laborde; } \\
\text { 1935: J. Brunhes } \\
\text { Cours moyen: } \\
\text { 1882: E. Levasseur; } \\
\text { 1891: H. Lemonnier, F. } \\
\text { Schrader; 1910: L.E. } \\
\text { Rogier, P. Despiques; } \\
\text { 1921: H. Lemonnier, F. } \\
\text { Schrader; 1925: P. } \\
\text { Kaepplin; 1926: R. } \\
\text { Blanchard, D. Faucher; } \\
\text { 1930: J. Brunhes; 1947 } \\
\text { : R. Clozier, P. } \\
\text { Fénelon; 1955: L. } \\
\text { Planel; 1957: A. Mer- } \\
\text { lier, G. Leroux. }\end{array}$ & $\begin{array}{l}\text { 1899: Frères de l'école } \\
\text { chrétienne; 1910: P. } \\
\text { Despiques; 1911: M. } \\
\text { Dubois, E. Sieurin; } \\
\text { 1914: H. Lemonnier, F. } \\
\text { Schrader; 1914: L. Gal- } \\
\text { louédec, F. Maurette; } \\
\text { 1924: L. Gallouédec, F. } \\
\text { Maurette; 1932: L. Gal- } \\
\text { louedec, F. Maurette; } \\
\text { 1933: P. Kaeppelin, M. } \\
\text { Teissier; 1933: M. Fal- } \\
\text { lex, A. Gibert, R. } \\
\text { Ozouf. }\end{array}$ & $\begin{array}{l}\text { Enseignement de jeunes filles: } \\
\text { 1885: M.H. Pigeonneau; 1911: M. Dubois, E. Sieurin } \\
\text { Troisième: } \\
\text { 1889: P. Foncin; 1908: M. Fallex, A. Mairey; 1945: P. } \\
\text { Hallynck, A. Ferré; 1947: H. Boucau, J. Petit, A. Leyritz; } \\
\text { 1953: F. Pinardel; 1954: P. Hallynck, A. Ferré } \\
\text { Première: } \\
\text { 1897, 1902 et 1910: P. Vidal de la Blache, P. Camena } \\
\text { d'Almeida; 1903: F. Schrader, L. Gallouédec; 1929: L. } \\
\text { Gallouédec, F. Maurette; 1931: M. Auzelet; 1933: M. } \\
\text { Fallex; 1934: F. Pinardel; 1935: H. Boucau; 1937: E. } \\
\text { Baron; 1942: L. François, R. Mangin; 1946: H. Boucau; } \\
\text { 1947: E. Baron; 1949: A. Gibert, G. Turlot; 1950: P. } \\
\text { Hallynck, R. Lugand; 1954: H. Boucau, J. Petit; 1957: P. } \\
\text { Hallynck, A. Ferré; 1959: W. Diville; 1959: cours } \\
\text { Varon; 1960: H. Jeanblanc, E. Verdier; 1961: G. Toussaint; } \\
\text { 1961: J. Pelletier, J. Virlogeux; 1962: P. Gourou, L. } \\
\text { Papy; 1963: J.P. Moreau, J. Ozouf; 1963: M. Baleste; } \\
\text { 1963: R. Blanchon, A. Labaste, R. Oudin; 1963: J. Madre; } \\
\text { 1963: V. Prévot; 1963: R. Guglielmo, Y. Lacoste, M. } \\
\text { Ozouf; 1964: J.P. Allix; 1966: A. Blanc, L. Pernet; } \\
\text { 1966: M. Le Lannou; 1967: A. Labaste; 1968: V. Prévot, } \\
\text { W. Diville; 1972: A. Labaste, M. Baleste: 1973: Y. } \\
\text { Lacoste; 1973: A. Labaste, M. Baleste; 1973: L. Pernet. }\end{array}$ \\
\hline
\end{tabular}

